\title{
Novel gain of function approaches for vaccine candidate identification in Burkholderia pseudomallei
}

\author{
Andrea J. Dowling* \\ Biosciences, College of Life and Environmental Sciences, University of Exeter, Penryn, UK
}

Edited by:

Mark Estes, University of Georgia, USA

\section{Reviewed by:}

Xiao-Lian Zhang, Wuhan Medical School, China

Yongqun He, University of Michigan School of Medicine, USA

*Correspondence:

Andrea J. Dowling, Biosciences,

College of Life and Environmental

Sciences, University of Exeter in

Cornwall, Tremough Campus,

Penryn TR10 9EZ, UK.

e-mail: a.j.dowling@exeter.ac.uk
The Gram-negative bacterium Burkholderia pseudomallei is a serious environmental pathogen and the causative agent of the often fatal melioidosis. Disease occurs following exposure to contaminated water or soil, usually through cuts in the skin or via inhalation. However, the underlying mechanisms of pathogenicity remain poorly understood. B. pseudomallei is endemic to South East Asia and Northern Australia where infections are associated with antibiotic resistance and high mortality rates. Categorization of the pathogen as a potential biowarfare agent has also made research into vaccine development a high priority. Recent genome-scale screening has produced a large number of putative gene candidates from B. pseudomallei with the potential for development into vaccines. This mini-review will discuss the advantages and limitations of this novel approach, how these new techniques can complement existing strategies, and outline aims for future research.

Keywords: Burkholderia, vaccine, melioidosis, novel screening, virulence factors

\section{INTRODUCTION}

Burkholderia pseudomallei is a serious environmental pathogen of man and the causative agent of the often fatal disease melioidosis. Infection occurs following exposure to contaminated water or soil, usually through cuts in the skin or via inhalation, but the underlying mechanisms of pathogenicity of B. pseudomallei to humans remain poorly understood (Adler et al., 2009). B. pseudomallei is endemic to South East Asia and Northern Australia where infections are associated with both antibiotic resistance and high patient mortality $(\sim 50 \%)$. The high rates of infection and subsequent mortality make $B$. pseudomallei a high priority for research and vaccine development, as no effective vaccine currently exists. A combination of the lack of vaccine, high infection rate via aerosol inhalation, and limited utility of antibiotics has resulted in classification of this bacterium as a Category B biowarfare pathogen (Rotz et al., 2002).

During the establishment of infection B. pseudomallei adheres to, survives and replicates within both host epithelial cells and macrophages. The bacterium is therefore capable of interfering with the host cellular mechanisms that would otherwise destroy it. Known bacterial factors affecting this interaction with host cells include the bacterial capsule, and effectors delivered by the type III and type VI secretion systems (T3SS and T6SS) (Stevens et al., 2003; Shalom et al., 2007; Galyov et al., 2010; Burtnick et al., 2011). Once inside the macrophage the pathogen induces cell fusion leading to the formation of "Multi-Nucleated Giant Cells" or MNGCs, a process key to both intracellular replication and bacterial persistence (Kespichayawattana et al., 2000). Following the invasion of host cells pathogen replication reaches a critical point at which the bacteria induce cell death and are released to establish secondary infections (Adler et al., 2009). Both the intrinsic drug resistance and the intracellular lifestyle of $B$. pseudomallei make successful antibiotic treatment difficult therefore vaccine development is an important strategy against melioidosis.

\section{CHALLENGING MACROPHAGES TO IDENTIFY ANTI-IMMUNE CELL FACTORS}

Evidence suggests that macrophages are an essential early defence against Burkholderia infection (Breitbach et al., 2006). These innate immune cells are one of the first responding phagocytes to arrive at the site of infection where they phagocytose invading bacteria and (along with other cells) release chemokines and cytokines to govern immune responses and promote adaptive immunity. To establish intracellular infection Burkholderia must evade macrophage killing and disrupt the normal cell signaling and regulation processes. In order to detect potential vaccine candidates a simple "gain of function" screen was designed aimed at identifying genes encoding anti-macrophage virulence factors across the B. pseudomallei genome (Figure 1). This screen identifies recombinantly expressed genes that equip naïve Escherichia coli with anti-macrophage abilities in three different ways: (1) the production of cytotoxic moieties e.g., proteins, secondary metabolites (toxins, effectors), (2) the ability to evade phagocytosis or destruction within the phagolysosome (intracellular survival), and (3) overwhelming e.g., nutrient depletion and starvation (biofilm formation, hydrogen peroxide scavenging) (Dowling et al., 2010).

\section{POTENTIAL VACCINE CANDIDATES IDENTIFIED}

A large proportion of melioidosis vaccines tested to date have focused on the use of live-attenuated strains of B. pseudomallei. However, a major issue with live-attenuated vaccines is the risk of reversion to virulence and although they are currently 


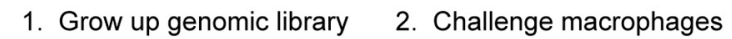

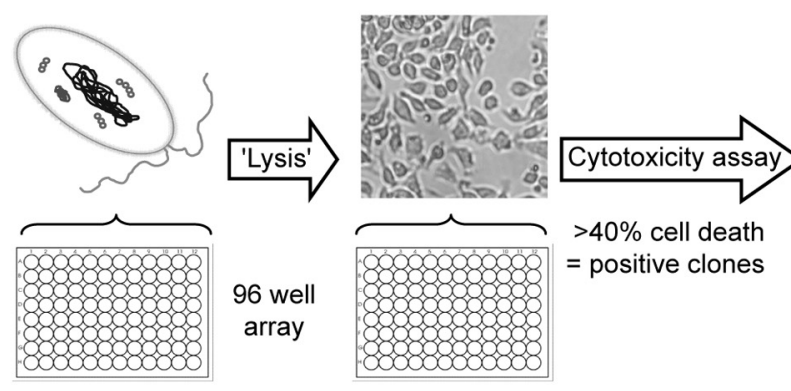

3. Map end-sequences of positive clones onto genome

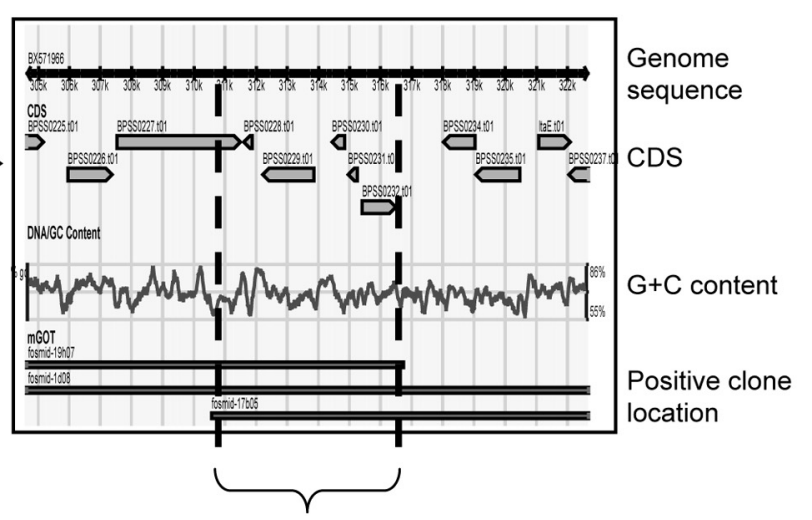

4. Minimum genetic overlap identifies ROI
FIGURE 1 | Brief overview of the anti-macrophage screen workflow. Recombinant genomic libraries of the sequenced clinical isolate B. pseudomallei K96243 were produced in E. coli (1), end-sequenced and subsequently screened for cytotoxicity against murine macrophages (2). Library clones shown to reduce macrophage viability by $40 \%$ or more are classed as positive and the end sequences of these positive clones are aligned onto the genome sequence (3). Each anti-macrophage locus, or region of interest (ROI), is then described as the minimum region of genetic overlap covered by two or more positive clones (4). the most effective vaccines studied in mice it is unlikely that they would be used in humans (Sarkar-Tyson and Titball, 2010). Less research has been focused on the development of subunit vaccines (mainly as a putative antigen needs to first be identified) and those investigated are far less effective (Patel et al., 2011). This screen represents a novel method that can be used to directly identify potential recombinant subunit vaccines. The design of the screen means that any active anti-macrophage products identified are successfully expressed within recombinant E. coli. Recombinant subunit vaccines are an attractive proposition as they eliminate the safety issues associated with subunit purification from large cultures of the pathogen itself (Liljeqvist and Stahl, 1999).

The original screen revealed over 100 anti-macrophage related loci on both chromosomes 1 and 2 of B. pseudomallei strain K96243. Several functional classes were repeatedly identified; putative secondary metabolite synthesis gene clusters (NRPSs and PKSs), signal response and regulation systems (methylaccepting chemotaxis proteins, two-component sensor systems, AraC and LysR family regulators), phage-related elements, resistance (drugs, metals, reactive oxygen species), adhesion and biofilm formation. The largest number of coding sequences (CDSs) detected however related to toxin/enzyme production (hemolysins, proteases, phospholipases) or transport and secretion (ABC transporters, autotransporters, efflux transporters, and components of Type III and Type VI secretion systems). Examples of putative vaccine candidates from different functional classes discussed in the following text are summarized in Table 1.

\section{TRANSPORTERS AND SECRETION SYSTEMS}

$\mathrm{ABC}$ transporter and autotransporter-related proteins are drawing considerable interest as potential vaccine candidates given the surface association of their component proteins which may be exposed to the immune system upon B. pseudomallei infection (Garmory and Titball, 2004; Lazar Adler et al., 2011). ABC transporters have a known role in bacterial survival and pathogenicity, 22 of the 105 predicted K96243 ABC transporters were detected by the screen (Harland et al., 2007b; Dowling et al., 2010). Of particular interest among the detected loci are the lipopolysaccharide (LPS) biosynthesis operon and wzt ABC transporter (Table 1). This particular finding suggests that an active LPS can be successfully constructed in a recombinant system. Given that LPS elicits strong immune responses this could make it an attractive vaccine candidate. Further, this screen detected 5 out of the 11 documented autotransporters in K96243 and interestingly detects both BPSS0796 (BoaA) and BPSL1705 (BoaB), these trimeric autotransported adhesins are implicated in adhesion to epithelial cells and are shown to have immunogenic properties (Tiyawisutsri et al., 2007; Balder et al., 2010).

Recent advances have been made regarding Type VI secretion machinery proteins; in particular the Hcp family, as subunit vaccine candidates (Burtnick et al., 2011). Three of the six documented hcp encoded proteins; Hcp5 (BPSS099), Hcp4 (BPSS0171), and Hcp6 (BPSL3015), were within ROI detected by this screen. However, research so far demonstrates that purified Hcp2 gives the highest-level protection (80\%) to BALB/C mice inoculated with a lethal dose of B. pseudomallei. Burtnick et al. demonstrated T6SS 1 as a major virulence determinant for $B$. pseudomallei with deletion of $h c p 1$ resulting in higher $\mathrm{LD}_{50 \mathrm{~s}}$ and growth defects compared to wild-type $B$. pseudomallei. Deletion of $\operatorname{vgr} 1$ from this system also displayed the same results demonstrating its importance in secretion of Hcpl (Burtnick et al., 2011). VgrG proteins play an important role in host cell cytotoxicity as well as forming the secretion machinery itself and are present on the surface of the pathogen, several VgrG CDSs were identified by the screen (Pukatzki et al., 2009; Suarez et al., 2010). 
Table 1 | Examples of putative vaccine candidates identified.

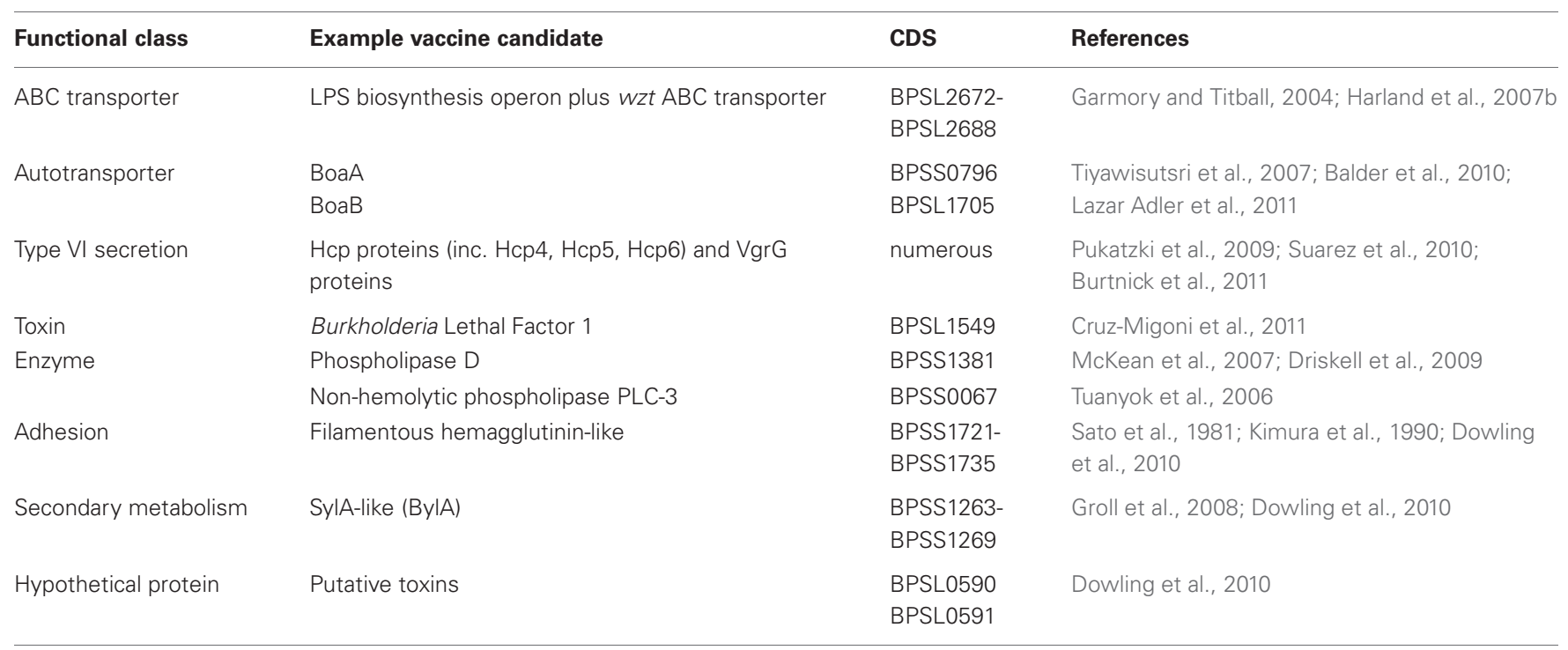

\section{TOXINS, ENZYMES, AND HYPOTHETICAL PROTEINS}

One of the anti-macrophage regions identified contains a putative Phospholipase D-like protein (BPSS1381) (Dowling et al., 2010). Mutations of Phospholipase D proteins in the pathogens Corynebacterium pseudotuberculosis and Rickettsia prowazekii, in which the protein is a major virulence factor, have shown potential as live-attenuated vaccines (McKean et al., 2007; Driskell et al., 2009). Further characterization is warranted to determine if this phospholipase D-like protein may be of use as a potential vaccine candidate for $B$. pseudomallei. Another loci of interest encodes the putative toxin PLC-3 (non-hemolytic phospholipase) which has been shown to be strongly upregulated in a hamster model of melioidosis and a mutant displays an $\mathrm{LD}_{50}$ significantly higher than wild-type B. pseudomallei parent strain (Tuanyok et al., 2006). These data highlight PLC-3 as a potential vaccine candidate warranting further investigation.

Approximately $30 \%$ of $B$. pseudomallei genes encode hypothetical proteins whose biological functions are yet to be established. These hypothetical proteins are a potential source of novel virulence factors, and indeed potential vaccine candidates. The anti-macrophage screen promotes the identification of hypothetical proteins with anti-macrophage functions. One positive region described by the screen contained two large adjacent CDSs, BPSL0590 and BPSL0591, encoding hypothetical proteins. The position-specific-iterative BLAST (psi-BLAST) algorithm predicted homology within BPSL0590 and BPSL0591 to known toxins from other pathogens. Both contain domains with homology to the Toxin complexes (Tc's) from Photorhabdus, further the predicted N-terminal of BPSL0590 has homology to the Salmonella enterica virulence associated protein or SpvB. Macrophages treated with lysate from recombinant E. coli library clones expressing BPSL0590 and BPSL0591 show altered actin cytoskeletal morphology, multi-nucleation, and apoptosis illustrating that these may be novel toxins (Dowling et al., 2010).

Recently a novel toxin has been described in B. pseudomallei K96243 with similarity to the potent CNF1 toxin from E. coli and has been named "Burkholderia Lethal Factor 1" or BLF1 (BPSL1549) (Cruz-Migoni et al., 2011). BLF1 has the ability to inhibit helicase activity and thus protein synthesis. BLF1 was previously described as a hypothetical protein given that similarity to CNF1 is based on structural rather than sequence homology. BLF1 mutants were attenuated in their ability to kill mice and purified toxin was lethal upon injection indicating the important nature of this toxin in B. pseudomallei virulence (Cruz-Migoni et al., 2011). The BPSL1549 CDS that encodes BLF1 was identified within a positive locus pulled out by the anti-macrophage screen. Of note, the toxin is flanked by two ABC transporter systems (BPSL1545-BPSL1546 and BPSL1548) also contained with the anti-macrophage region of interest that may be involved in secretion of BLF1. The utility of BLF1 as a toxoid vaccine has yet to be explored. There are a further 28 anti-macrophage loci out of the 113 described in the screen which contain at least one hypothetical protein. These are yet to be investigated via detailed protein and structural prediction algorithms to identify active sites or homologies. However, as exemplified by BLF1, such hypothetical proteins warrant further research as a source of potential, currently hidden, virulence factors, and hopefully future vaccine candidates.

\section{ADHESINS}

Adhesins make attractive vaccine candidates, as they are surface expressed and likely therefore to be presented to the immune system upon infection. Adhesins are often key virulence determinants allowing the initial attachment of the invading pathogen to host cells. The effectiveness of the Filamentous hemagglutinin (FHA) of Bordetella pertussis as an antigen has long been utilized in vaccines against Pertussis (Sato et al., 1981). The region BPSS1721-BPSS1735 identified as an anti-macrophage locus encodes proteins with predicted homology to B. pertussis FHA. Macrophages treated with library clones expressing the region showed dramatic actin alterations and apoptotic nuclei. Of note, $B$. pertussis FHA is successful at preventing adherence 
of the pathogen to the respiratory epithelium when introduced as a protective antigen via intranasal immunization (Kimura et al., 1990). The role of this hemagglutinin-like protein in B. pseudomallei infection has not so far been characterized. Determination of whether this FHA homolog may prove useful in protection against melioidosis, in particular disease contracted via inhalation, could help in the formulation of a successful vaccine.

\section{SECONDARY METABOLITES}

Many of the anti-macrophage regions identified contained large gene clusters associated with the non-ribosomal peptide synthetase (NRPS) or polyketide synthase (PKS) systems used in the production of peptides and small molecules. The role of such secondary metabolites in virulence is largely undescribed, however, they may be used to promote intracellular survival or production of toxic products and could provide part of the underlying metabolism supporting latent disease. One such anti-macrophage region described by the screen encodes an NRPS gene cluster (BylA) with homology to the SylA (Syringolin A) producing genes from Pseudomonas syringae (Dowling et al., 2010). SylA is a proteasome inhibitor and potent cytotoxin (Groll et al., 2008). NRPS and PKS gene clusters and their secondary metabolite products are an unexplored class of vaccine candidates. Purified secondary metabolites may be directly useful as antigens, and there is also the potential for mutating NRPS/PKS gene for use as live-attenuated vaccines.

\section{PREVIOUSLY EVALUATED VACCINE CANDIDATES IDENTIFIED BY THE SCREEN}

The subunit vaccine candidate PotF and the asd (aspartate- $\beta$ semialdehyde dehydrogenase) gene whose mutation has been investigated for use as a live-attenuated vaccine were re-identified (Harland et al., 2007a; Norris et al., 2011). Asd amino acid products have a role in cell wall biosynthesis and are likely to be necessary for growth and replication (Norris et al., 2011). The asd gene may have equipped recombinant library E. coli with an increased growth ability enabling them to cause cytotoxicity by overwhelming the macrophages. PotF is an ABC transporterassociated protein involved in putrescine transport documented as necessary for virulence in other bacterial pathogens, putatively involved in host cell attachment and virulence as in a homologous ABC transporter in the plant pathogen Agrobacterium tumefaciens and for invasion and intracellular survival in Salmonella enterica (Matthysse et al., 1996; Jelsbak et al., 2012). Outer membrane protein A (OmpA) members Omp3 and Omp7 (Hara et al., 2009) and also Omp85 (Su et al., 2010) have been researched as subunit vaccine candidates, however, protection afforded was no greater than with other strategies. Several Omps were identified by this screen, but not those so far tested as candidates. Interestingly, the majority of previously studied vaccine candidates were not re-identified. The main reason for this is that the screen looks specifically for toxicity toward macrophages. Existing subunit candidates were selected for potential immunogenicity and are not necessarily cytotoxic. Most of the genes selected for mutation in the development of live-attenuated vaccines are involved in biosynthetic processes and expression of these in library strain E. coli may not result in production of cytotoxic moieties or cytotoxicity as a result of enhanced bacterial growth and overwhelming.

\section{ADVANTAGES AND LIMITATIONS}

There are several advantages of using gain of function screens for discovering virulence factors and vaccine candidates. Firstly, they overcome functional redundancy to unmask virulence factors which may be compensated in mutants. B. pseudomallei has a large genome and the ability to survive in a wide range of environmental niches. This suggests that functional redundancy is likely among virulence factors with various genes or genetic regions able to compensate mutation to produce successful infection. Secondly, the screens cannot only be used to directly isolate candidates, which may be useful in the development of recombinant subunit vaccines, but also subunit vaccines, DNA vaccines, and live-attenuated vaccines. The screen works by identifying factors that are effective against immune cells via successful recombinant expression in E. coli. Production of vaccines via recombinant expression is safer, and more efficient, than purifying from the pathogen itself. Further, the technique narrows down the minimum genetic area needed to produce a response. So far purified proteins have had limited success as vaccines, mainly as this approach requires actual identification of virulence factors or antigenic determinants as candidates first. This problem is tackled by the screening process, which is also able to identify hypothetical proteins with a role in macrophage toxicity. Identifying virulence factors and/or antigens has previously proved difficult for intracellular bacteria (Titball, 2008; Patel et al., 2011). We are currently developing a further novel genomic screen to look for virulence factors involved in intracellular survival and persistence. Thirdly, such screening is rapid and cost-effective, without needing Class III safety facilities and trained staff. Such requirements can restrict the progress of vaccine candidate identification and testing.

One limitation of the screen is the size range of DNA fragments cloned into the BAC and fosmid libraries used $(10 \mathrm{~kb}$ and $40 \mathrm{~kb}$ respectively) therefore any factors above this size will be missed (for example complete type III secretion systems). For successful screening high library quality, in particular good genomic coverage, is necessary to allow repeated identification of cytotoxic clones containing the same genetic region in order to generate confidence in the ROIs identified. The E. coli used in screens to date are not modified with for example, regulatory systems, functional secretion systems, or promoters of secondary metabolite production. Although this may limit what is detected, it is also serves as an advantage, as any cytotoxic activity detected is simply a result of successful production by the recombinant library E. coli expressing a $B$. pseudomallei DNA fragment. This immediately allows us to narrow down what can be produced in a recombinant system and importantly identify the minimum genetic region necessary to produce bioactivity in such a recombinant system, as would be required for culture and production of a recombinant subunit vaccine. Successful secretion/release from recombinant E. coli and effector uptake by macrophages may also limit the factors detected. Some factors may achieve access to macrophages as the cells take in solutes non-specifically from the extracellular 
media (inoculated with clone lysate preparation) via pinocytosis/macropinocytosis. Further, some viable E. coli in the lysate preparation appears necessary for toxicity in some cases.

\section{PATHWAYS FOR FUTURE RESEARCH}

The number of candidates identified by the gain of function screen is large $(>100)$. Combined approaches correlating datasets obtained from techniques such as in vivo expression with this type of recombinant screen will further narrow down key vaccine candidates for follow-up. Use of advanced cytotoxicity screens and high-content phenotype screening will allow detailed functional characterization of candidates. Further investigation of positive regions identified will involve transposon mutagenesis to identify minimum region of activity/genes involved and sub-cloning of these genes to confirm activity. Another useful next step would be to measure the extent of the immune response of macrophages challenged with the anti-macrophage factors identified from the original screen by, for example, assaying nitric oxide production. Screening for adherence and intracellular persistence will identify factors involved in these aspects of $B$. pseudomallei infection. This could prove valuable for development of live-attenuated vaccines for which the issues of safety and potential latency of the mutant B. pseudomallei bacteria are of major concern. Introducing additional mutations which will limit or prevent adherence to or

\section{REFERENCES}

Adler, N. R., Govan, B., Cullinane, M., Harper, M., Adler, B., and Boyce, J. D. (2009). The molecular and cellular basis of pathogenesis in melioidosis: how does Burkholderia pseudomallei cause disease? FEMS Microbiol. Rev. 33, 1079-1099.

Balder, R., Lipski, S., Lazarus, J. J., Grose, W., Wooten, R. M., Hogan, R. J., et al. (2010). Identification of Burkholderia mallei and Burkholderia pseudomallei adhesins for human respiratory epithelial cells. BMC Microbiol. 10:250. doi: 10.1186/1471-2180-10-250

Breitbach, K., Klocke, S., Tschernig, T., Van Rooijen, N., Baumann, U., and Steinmetz, I. (2006). Role of inducible nitric oxide synthase and NADPH oxidase in early control of Burkholderia pseudomallei infection in mice. Infect. Immun. 74, 6300-6309.

Burtnick, M. N., Brett, P. J., Harding, S. V., Ngugi, S. A., Ribot, W. J., Chantratita, N., et al. (2011). The cluster 1 type VI secretion system is a major virulence determinant in Burkholderia pseudomallei. Infect. Immun. 79, 1512-1525.

Cruz-Migoni, A., Hautbergue, G. M., Artymiuk, P. J., Baker, P. J., BokoriBrown, M., Chang, C. T., et al. (2011). A Burkholderia pseudomallei toxin inhibits helicase activity of translation factor eIF4A. Science 334, 821-824.

Dowling, A. J., Wilkinson, P. A., Holden, M. T., Quail, M. A., Bentley, S. D., Reger, J., et al. (2010). Genome-wide analysis reveals loci encoding antimacrophage factors in the human pathogen Burkholderia pseudomallei K96243. PLoS ONE 5:e15693. doi: 10.1371/journal.pone.0015693

Driskell, L. O., Yu, X. J., Zhang, L., Liu, Y., Popov, V. L., Walker, D. H., et al. (2009). Directed mutagenesis of the Rickettsia prowazekii pld gene encoding phospholipase D. Infect. Immun. 77, 3244-3248.

Galyov, E. E., Brett, P. J., and Deshazer, D. (2010). Molecular insights into Burkholderia pseudomallei and Burkholderia mallei pathogenesis. Annu. Rev. Microbiol. 64, 495-517.

Garmory, H. S., and Titball, R. W. (2004). ATP-binding cassette transporters are targets for the development of antibacterial vaccines and therapies. Infect. Immun. 72, 6757-6763.

Groll, M., Schellenberg, B., Bachmann, A. S., Archer, C. R., Huber, R., Powell, T. K., et al. (2008). A plant pathogen virulence factor inhibits the eukaryotic proteasome by a novel mechanism. Nature 452, 755-758.

Hara, Y., Mohamed, R., and Nathan, S. (2009). Immunogenic Burkholderia

persistence within cells in the host could help to make this type of vaccine more viable for use (Patel et al., 2011). These gain of function screens can be used to "mix and match" recombinant vaccine candidates to investigate combinations for possible multivalent subunit vaccines. A combination vaccine composed of polysaccharide and protein presents the most promising formulation for a melioidosis vaccine to date (Patel et al., 2011).

\section{CONCLUDING REMARKS}

By directly asking macrophages which genomic regions of $B$. pseudomallei express anti-macrophage gene products in a recombinant library system we can begin to isolate virulence factors and potential vaccine candidates. We can explore and characterize the effects of single virulence factors provided that activity can be reproduced in recombinant E. coli. This "gain of function" technique can be strengthened by combining with other methods, for example, in vivo expression data, providing a powerful tool for discovery, and prioritization of key vaccine candidates for future research.

\section{ACKNOWLEDGMENTS}

I would like to thank all of those involved in the development of the screening process and Prof. R. ffrench-Constant for critical reading of the manuscript.

pseudomallei outer membrane proteins as potential candidate vaccine targets. PLoS ONE 4:e6496. doi: 10.1371/journal.pone.0006496

Harland, D. N., Chu, K., Haque, A., Nelson, M., Walker, N. J., Sarkar-Tyson, M., et al. (2007a). Identification of a LolC homologue in Burkholderia pseudomallei, a novel protective antigen for melioidosis. Infect. Immun. 75 , 4173-4180.

Harland, D. N., Dassa, E., Titball, R. W., Brown, K. A., and Atkins, H. S. (2007b). ATP-binding cassette systems in Burkholderia pseudomallei and Burkholderia mallei. BMC Genomics 8:83. doi: 10.1186/14712164-8-83

Jelsbak, L., Thomsen, L. E., Wallrodt, I., Jensen, P. R., and Olsen, J. E. (2012). Polyamines are required for virulence in Salmonella enterica serovar Typhimurium. PLoS ONE 7:e36149. doi: 10.1371/journal.pone.0036149

Kespichayawattana, W., Rattanachetkul, S., Wanun, T., Utaisincharoen, P., and Sirisinha, S. (2000). Burkholderia pseudomallei induces cell fusion and actinassociated membrane protrusion: a possible mechanism for cell-tocell spreading. Infect. Immun. 68, 5377-5384.

Kimura, A., Mountzouros, K. T., Relman, D. A., Falkow, S., and Cowell, J. L. (1990). Bordetella pertussis filamentous hemagglutinin: evaluation as a protective antigen and colonization factor in a mouse respiratory infection model. Infect. Immun. 58, 7-16.

Lazar Adler, N. R., Stevens, J. M., Stevens, M. P., and Galyov, E. E. (2011). Autotransporters and their role in the virulence of Burkholderia pseudomallei and Burkholderia mallei. Front. Microbiol. 2:151. doi: 10.3389/fmicb.2011.00151

Liljeqvist, S., and Stahl, S. (1999). Production of recombinant subunit vaccines: protein immunogens, live delivery systems and nucleic acid vaccines. J. Biotechnol. 73, 1-33.

Matthysse, A. G., Yarnall, H. A., and Young, N. (1996). Requirement for genes with homology to $\mathrm{ABC}$ transport systems for attachment and virulence of Agrobacterium tumefaciens. J. Bacteriol. 178, 5302-5308.

McKean, S. C., Davies, J. K., and Moore, R. J. (2007). Expression of phospholipase $\mathrm{D}$, the major virulence factor of Corynebacterium pseudotuberculosis, is regulated by multiple environmental factors and plays a role in macrophage death. Microbiology 153, 2203-2211.

Norris, M. H., Propst, K. L., Kang, Y., Dow, S. W., Schweizer, H. P., and Hoang, T. T. (2011). The Burkholderia pseudomallei Deltaasd mutant exhibits attenuated intracellular infectivity and imparts protection against acute inhalation 
melioidosis in mice. Infect. Immun. 79, 4010-4018.

Patel, N., Conejero, L., De Reynal, M., Easton, A., Bancroft, G. J., and Titball, R. W. (2011). Development of vaccines against Burkholderia pseudomallei. Front. Microbiol. 2:198. doi: 10.3389/fmicb.2011.00198

Pukatzki, S., McAuley, S. B., and Miyata, S. T. (2009). The type VI secretion system: translocation of effectors and effector-domains. Curr. Opin. Microbiol. 12, 11-17.

Rotz, L. D., Khan, A. S., Lillibridge, S. R., Ostroff, S. M., and Hughes, J. M. (2002). Public health assessment of potential biological terrorism agents. Emerg. Infect. Dis. 8, 225-230.

Sarkar-Tyson, M., and Titball, R. W. (2010). Progress toward development of vaccines against melioidosis: a review. Clin. Ther. 32, 1437-1445.

Sato, Y., Izumiya, K., Sato, H., Cowell, J. L., and Manclark, C. R. (1981).
Role of antibody to leukocytosispromoting factor hemagglutinin and to filamentous hemagglutinin in immunity to pertussis. Infect. Immun. 31, 1223-1231.

Shalom, G., Shaw, J. G., and Thomas, M. S. (2007). In vivo expression technology identifies a type VI secretion system locus in Burkholderia pseudomallei that is induced upon invasion of macrophages. Microbiology 153, 2689-2699.

Stevens, M. P., Friebel, A., Taylor, L. A., Wood, M. W., Brown, P. J., Hardt, W. D., et al. (2003). A Burkholderia pseudomallei type III secreted protein, BopE, facilitates bacterial invasion of epithelial cells and exhibits guanine nucleotide exchange factor activity. J. Bacteriol. 185, 4992-4996.

Su, Y. C., Wan, K. L., Mohamed, R., and Nathan, S. (2010). Immunization with the recombinant Burkholderia pseudomallei outer membrane protein Omp85 induces protective immunity in mice. Vaccine 28, 5005-5011.

Suarez, G., Sierra, J. C., Erova, T. E., Sha, J., Horneman, A. J., and Chopra, A. K. (2010). A type VI secretion system effector protein, VgrG1, from Aeromonas hydrophila that induces host cell toxicity by ADP ribosylation of actin. J. Bacteriol. 192, 155-168.

Titball, R. W. (2008). Vaccines against intracellular bacterial pathogens. Drug Discov. Today 13, 596-600.

Tiyawisutsri, R., Holden, M. T., Tumapa, S., Rengpipat, S., Clarke, S. R., Foster, S. J., et al. (2007), Burkholderia Hep_Hag autotransporter (BuHA) proteins elicit a strong antibody response during experimental glanders but not human melioidosis. BMC Microbiol. 7:19. doi: 10.1186/1471-2180-7-19

Tuanyok, A., Tom, M., Dunbar, J., and Woods, D. E. (2006). Genome-wide expression analysis of Burkholderia pseudomallei infection in a hamster model of acute melioidosis. Infect. Immun. 74, 5465-5476.

Conflict of Interest Statement: The author declares that the research was conducted in the absence of any commercial or financial relationships that could be construed as a potential conflict of interest.

Received: 05 July 2012; accepted: 22 October 2012; published online: 09 January 2013.

Citation: Dowling AJ (2013) Novel gain of function approaches for vaccine candidate identification in Burkholderia pseudomallei. Front. Cell. Inf. Microbio. 2:139. doi: 10.3389/fcimb.2012.00139 Copyright (c) 2013 Dowling. This is an open-access article distributed under the terms of the Creative Commons Attribution License, which permits use, distribution and reproduction in other forums, provided the original authors and source are credited and subject to any copyright notices concerning any thirdparty graphics etc. 